\title{
Interviewing Over Instant Messaging
}

\author{
Amy Voida \& Elizabeth D. Mynatt \\ College of Computing \\ GVU Center, Georgia Tech \\ Atlanta, GA, 30332 \\ \{amyvoida, mynatt\}@cc.gatech.edu
}

\author{
Thomas Erickson \& Wendy A. Kellogg \\ IBM T.J. Watson Research Center \\ P.O. Box 704 \\ Yorktown Heights, NY 10598 \\ \{snowfall,wkellogg\}@us.ibm.com
}

\begin{abstract}
Interviews are a cornerstone of human-computer interaction research. As a research method, they can both be deeply valuable and distinctly challenging. Pragmatic challenges of interviews include the travel that may be required to meet face-to-face with a respondent or the time necessary to transcribe the exchange. As a tool for conducting interviews, instant messaging presents some compelling potential benefits to mitigate challenges such as these. And yet, over the medium of instant messaging, the genre of the interview takes on a different character. Drawing from our experiences conducting interviews over instant messaging, we reflect on the implications of using this new medium for conducting interviews.
\end{abstract}

\section{Author Keywords}

Interviewing, instant messaging, qualitative research methods, computer-mediated communication, genre

\section{ACM Classification Keywords}

I.m [Computing Methodologies]: MiscellaneousInterviewing; H.4.3 [Information Systems Applications]: Communications Applications

\section{INTRODUCTION}

Interviews are a cornerstone of human-computer interaction research. As a research method, they can both be deeply valuable and distinctly challenging. Pragmatic challenges of interviews include the travel that may be required to meet face-to-face with a respondent or the time necessary to transcribe the exchange. As a tool for conducting interviews, instant messaging presents some compelling potential benefits to mitigate challenges such as these. And yet, over the medium of instant messaging, the genre of the interview takes on a different character.

Over the course of approximately two and a half weeks, we conducted sixteen interviews over instant messaging (IM). In our case, one very practical motivation for using IM was that some of our respondents were not employed locally and traveling to interview them in person was not an option. Another practical reason for this was that we were under rather severe time constraints and the time it would have taken to transcribe the interviews would otherwise have been prohibitive. In addition, the purpose of the interviews was to explore various issues related to privacy and IM; we were interested in whether the use of IM for a purpose that might have privacy implications would elicit any relevant meta-data.

In this paper, we briefly describe both the interview genre and the instant messaging medium. Given those descriptions, we reflect on ways that aspects of instant messaging impact interviewing, from expectations about attention while using instant messaging to its timing, limited context and persistence.

\section{The Interview Genre}

The typical interview is usually a dyadic interaction and usually happens face-to-face. Because of this, social expectations are such that the interviewer and the respondent are likely to be fully focused on the activity of the interview. Also because of this, the parties are generally privy to conversational context, including non-verbal communication such as body language and tone of voice. Beforehand, the interviewer generally prepares topics of interest or open-ended questions from which to draw in the interview. During the interview, a balance between too much and too little rapport with the respondent is established. Through presence and eye-contact, for example, the interviewer may provide subtle cues that what the respondent is saying is both useful and interesting. The interviewer probably takes notes and may also be recording what the respondent has to say. The bulk of the talking is generally done by the respondent; the interviewer will usually interrupt as little as possible as long as the respondent hasn't strayed too far from the intent of the interview. In general, the respondent develops an answer to a question over the course of talking and may shift the focus of the answer after having thought through the question out loud $[3,5]$.

The interview may be considered a genre in the sense that:

\footnotetext{
"Genres (e.g. the memo, the proposal, and the meeting) are typified communicative actions characterized by similar substance and form and taken in response to recurrent situations. These genres evolve over time in reciprocal interactions between institutionalized practices and individual human actions. They are distinct from communication media..." [6].
}

\section{The Instant Messaging Medium}

The medium of instant messaging supports nearsynchronous communication among two or more parties. It allows users to share ideas across long distances almost 
instantly and provides an environment that encourages fluid, rapid exchanges. Most IM clients display a persistent copy of the ongoing conversation until the conversation window is closed. Users edit new messages in a separate text box on their local machine; the contents of the instant message are updated when the users "post" their messages. Users may engage in multiple IM conversations at the same time or move between IM and other work tasks while their messaging partners read and prepare responses to their messages. In general, instant messaging also provides lightweight indications of awareness through usercontrolled status prompts and, in some clients, through user interface indications of whether remote users are actively writing a new comment in their instant messaging window $[1,2,4]$.

\section{IMPACT OF INSTANT MESSAGING ON INTERVIEWING}

"...media may play a role in genre form, and the introduction of new media may occasion genre evolution" [6].

Existing research on genre suggests that media impacts genre form. As such, instant messaging, as a medium, impacts the nature of the interview, as a genre. In the following discussion we reflect on how the experience of an otherwise typical interview changed, sometimes in striking ways, when carried out over a new medium instant messaging.

\section{Impact of Attention Expectations}

In our interviews, some of our respondents were unsure whether they should remain exclusively focused on the interview, as in a typical interview, or whether, as is more accepted within instant messaging, they were permitted to move the conversation in and out of focus. One of these respondents asked the interviewer if she was multitasking, presumably to gauge the appropriateness of doing so herself:

$$
\begin{aligned}
& \mathrm{R} 1^{1} \text { : Are you multi-task now? If you do, I'll do the } \\
& \text { same }^{2} \text {. }
\end{aligned}
$$

One of our interview "questions" involved asking the respondent to recount his or her last instant messaging encounter. To set the stage for that recollection, the interviewer asked the respondent when he or she last "IMed" someone. In one instance, the prompt elicited an admission that the respondent was engaged in another instant message at the same time that he was participating in the interview. The interesting thing to note here is that while it may normally be perfectly acceptable to carry on

${ }^{1}$ Our respondents are indicated by $R 1, R 2$, etc.

${ }^{2}$ In all excerpts, identifying information in the text has been anonymized, but idiosyncrasies of language and typographic errors have been preserved. more than one conversation over instant messaging at a given time, the respondent here felt the need to apologize for doing so under these particular circumstances:

$\mathrm{I}^{3}: \quad$ Before instant messaging with me, when was the
last time you IMed with someone?
$\mathrm{R} 2: \quad 10 \mathrm{~min}$ before
$\mathrm{R} 2: \quad$ and i have another going now...sorry!

As, perhaps, an extreme case, we also experienced one interview in which a respondent was drawn away from the interview for a full half hour before returning:

$\begin{array}{ll}\text { R3: } & \text { hold on } \\ \text { [approximately one half hour passes] } \\ \text { R3: } 1 \text { minute } \\ \text { R3: } \quad \text { SORRY! }\end{array}$

That some respondents felt comfortable multitasking during the interview did prove to be helpful on occasion. During one particular interview, the respondent raised possible issues that we had not anticipated. When we followed up for more information, the respondent was unable to verify her intuition. The respondent knew, however, just who to ask and started a second, concurrent instant message with that individual. In short order, she was able to verify information for our interview that allowed us to continue an interesting and unanticipated thread of inquiry.

We encountered another complication related to attention when attempting to provide appropriate feedback to our respondents during the interview process. In a typical interview, the interviewer can provide physical cues (e.g. presence and eye contact) that he or she is attending to what the respondent is saying. In a medium in which it is acceptable not to pay attention, it was frustrating not to be able to communicate that attentiveness to the respondent. Often, respondents would post series of long messages in slow succession. After one message was posted, a typing indicator would show that the respondent was continuing to type. As interviewers, we continued to "listen." But after lengthy series of postings, we began to wonder whether the respondents would think we had wandered off. In the end, when things felt unusually long and uncomfortable, we tried interspersing responses such as "uh huh" or "oh, I see" with the respondents' text. This was an unsatisfactory resolution, as the postings felt hollow and would have had the potential for distorting how the respondent answered [3]. In retrospect, this may not have been as much of an issue as we perceived; no respondent ever asked whether we were still attending to the conversation.

\section{Impact of Timing}

In instant messaging, because posted messages can be lengthy, it is both acceptable and necessary to take a minute after text has been posted to read and sometimes reflect

\footnotetext{
3 The interviewer is indicated by $I$.
} 
upon what was said; a response is not expected to be immediate. In fact, while conducting our interviews, it seemed to us rather rude and disrespectful to send a response too quickly following a respondent's posting.

For our interviews we had prepared a list of questions in advance and had the text of the questions available during the interview in a separate word processor window. As the questions became relevant, we often did some on-the-fly editing to tailor them to the particular context or tone of the interview before cutting and pasting them into the IM client. Sometimes, however, the original phrasing of the question did not require such a change. In one such case, the question was pasted into the IM client and was posted much more quickly than the respondent would have expected, given its length. The unusual timing of the post drew comment and was distracting to the flow of the interview:

R3: are you really typing this fast?

R3: $\quad$ or cuttng and pasting

The instant messaging medium also afforded the time for both parties to craft individual messages before sending them. We took advantage of this affordance to craft higherquality follow-up questions than we otherwise might have been able to ask. Due to the high quality of the responses we received during our interviews, we infer that our respondents were taking advantage of the same affordance to think through and refine their responses as well. However, this also meant that we would have no record of comments that they may have been writing and then selfcensoring before sending.

Because of the typing indicators in our instant messaging client, we were aware of instances when self-censoring was likely taking place. Typing indicators reflected that one respondent had spent approximately five minutes typing a continuation of a response to a question. When that response was posted, it only consisted of a three word question:

\section{R4: That's what I mean by condemning. [approximately five minutes passes] \\ R4: That make sense?}

Given a five-minute typing time, we assumed that the respondent had continued to elaborate and, at the last second, changed his mind, posting a question of confirmation that was likely significantly less rich than what had previously been typed.

Finally, timing implications of the use of instant messaging significantly changed the feel of the interviewing experience. Typical interviews are intense, consuming experiences; the mind is continually engaged with listening and understanding. The lag between messages meant that interviewing over instant messaging involved significantly more waiting. Initially, at least, there was a frustrating feeling of having one's hands tied, a level of detachment caused by spending so much time waiting for the next message. After the first few interviews, however, it became apparent that the lag between incoming responses could be viewed as a resource, affording time to look back at the current interview and remind oneself of respondents' comments to return to, to begin to synthesize across interviews, and also, in our case, to reflect and jot down notes about the experience of interviewing over instant messaging.

\section{Impact of Limited Context}

In interviewing, there is a fine balance to be struck between developing enough rapport with respondents and developing too much rapport with respondents [3]. The relative lack of context in instant messaging could have made initial rapport difficult to develop. However, the fact that instant messaging is often treated as a more informal medium seemed to make it easier to fall into a familiar, comfortable exchange.

Lack of context such as tone of voice and body language became most obvious as we tried to understand comments that the respondents perhaps felt should have been selfexplanatory. Instead of coming across as being genuinely interested in nuance or detail, the questions of the interviewer sometimes seemed to come across as being a reflection of slowness or stupidity and incited a certain amount of terseness, frustration, or perhaps crossness in one particular respondent (note the use of the interviewer's name, as if to scold her for asking an inappropriate or irritating question):

$$
\begin{array}{ll}
\text { R5: } & \text { Amy } \\
\text { R5: } & \text { The bottom line is } \\
\text { R5: } & \text { If they need what I tell them, they can save it all } \\
& \text { they want }
\end{array}
$$

\section{Impact of Persistence}

The primary anticipated benefit of instant messaging's persistence was the time savings in not having to transcribe the interviews. But, the persistence of instant messaging also brought with it some other surprising benefits.

Having a dynamically-generated transcript gave us a persistent source to refer to while unpacking specific comments that our respondents had made. It also allowed us to quote, via cut and paste, specific language that respondents had used previously in the conversation. We could scroll back to find a comment and ensure that the language we used was exactly the language that the respondent had used.

The persistence of instant messaging also enabled certain respondent behaviors that were unanticipated. Toward the end of all of our interviews, we asked the respondents if there were any other things they wanted to make sure they had the opportunity to tell us or whether there were any questions we should have asked that we did not. We have no explicit evidence, but it is feasible that the presence of a 
complete transcript could have allowed users to scroll back through the content of the interview to reflect on what was and was not actually said.

Similarly, in the particular series of interviews we conducted, we also asked the respondents whether there was anything they said that they would not be comfortable having shared with the research community. One respondent specified certain portions of the interview that she would prefer not be shared. Following the interview, we sent her a follow-up email asking how she had determined what comments should be excluded and it was in her reply that we came to understand the value of the persistence of instant messaging to the respondent:

\begin{abstract}
"I was using the scrollback feature to go back and look at what I'd said. I was trying to reinterpret my comments in light of potentially being published, rather than spoken directly to you in a one-on-one conversation. I was specifically looking for comments that violated other people's privacy (as in the [name of online community omitted] incident), or comments that could be misinterpreted when taken out of context.

It was really interesting to be hit with that question at the end. I should have expected it, given after all that it was a conversation about privacy in IM, but I didn't. During our conversation I was sharing information freely with you, as if I were talking to a colleague, but not as if talking to an interviewer. So the question came as a shock because it reminded me that the information I had shared with you was going to be made public contrary to my expectations. It's strange to me that I had such expectations."
\end{abstract}

\section{CONCLUSION}

Interviewing is a valuable method for human-computer interaction research and involves certain pragmatic challenges that may be mitigated by conducting interviews over instant messaging. That said, the character of the interview genre changed in sometimes significant ways when carried out over the instant messaging medium. We have reflected on our own experiences interviewing over instant messaging, exploring the ways in which expectations about attention, timing, limited context, and persistence impact the genre of the interview.

We will continue to experiment with the use of instant messaging for conducting interviews. Based on our experiences and observations, we intend to try some of the following strategies:

- asking respondents if they would be willing to share their prior thoughts or thought processes in the event that they type for several minutes and only send a short message;

- summarizing our understanding of what the respondents have said prior to asking follow-up questions in situations where probing for nuance could be perceived as slowness or a lack of understanding;

- being mindful of the pacing of the interview and the length of pre-written questions when cutting and pasting them into the interview; and

- taking advantage of instant messaging's persistence by explicitly suggesting that respondents scroll back and consider whether there was anything we had not discussed that they would like to add to the conversation.

By reflecting on our experiences interviewing over instant messaging and observing some interesting interactions between medium and genre, we hope to provide a variety of things to think about for those considering or planning to conduct interviews over instant messaging.

\section{ACKNOWLEDGMENTS}

Thanks to Ingrid Erickson and Stephen Voida for their thoughtful comments on this work. This research was conducted while the first author was a summer intern with the Social Computing Group at the IBM T.J. Watson Research Center.

\section{REFERENCES}

1. Isaacs, E., Walendowski, A., Whittaker, S., Schiano, D., \& Kamm, C. (2002). The character, functions, and styles of instant messaging in the workplace. In Proceedings of the ACM Conference on Computer Supported Cooperative Work. New York: ACM Press, pp. 11-20.

2. Nardi, B., Whittaker, S., \& Bradner, E. (2000). Interaction and outeraction: Instant messaging in action. In Proceedings of the ACM Conference on Computer Supported Cooperative Work. New York: ACM Press, pp. 79-88.

3. Seidman, I. (1998). Interviewing as qualitative research. New York: Teacher's College Press.

4. Voida, A., Newstetter, W.C., \& Mynatt, E.D. (2002). When conventions collide: The tensions of instant messaging attributed. In Proceedings of the ACM Conference on Human Factors in Computing Systems. New York: ACM Press, pp. 187-194.

5. Weiss, R.S. (1994). Learning from strangers: The art and method of qualitative interview studies. New York: The Free Press.

6. Yates, J. \& Orlikowski, W. (1992). Genres of organizational communication: A structurational approach to studying communication and media. Academy of Management Review, 17(5), 299-326. 